chimærism $^{3}$ and tolerance of homografts ${ }^{8}$ has long been known to be associated with infertility of the female. In our present experiments, tolerance has been conferred upon female mice by inoculation with male cells without affecting their fertility. The two phenomena are therefore separable, although in cattle they go together and share an anatomical pre-requisite in common; but this is an inference that leaves entirely open the question of whether or not the freemartin state is due to a purely humoral influence of the male fotus upon its synchorial female twin.

The experiments described in this article will in due course be reported on in full.

\section{Summary}

(1) Mice and chickens never develop, or develop to only a limited degree, the power to react immunologically against foreign homologous tissue cells with which they have been inoculated in foetal life. Animals so treated are tolerant not only of the foreign cells of the original inoculum, but also of skin grafts freshly transplanted in adult life from the original donor or from a donor of the same antigenic constitution.

(2) Acquired tolerance is immunologically specific : mice and chickens made tolerant of homografts from one donor retain the power to react against grafts transplanted from donors of different antigenic constitutions.
(3) Acquired tolerance is due to a specific failure of the host's immunological response. The antigenic properties of a homograft are not altered by residence in a tolerant host, and the host itself retains the power to give effect to a passively acquired immunity directed against a homograft which has until then been tolerated by it.

(4) The fertility of tolerant mice is unimpaired.

1 Cf. Rawles, M. E., Physiol. Rev., 28, 383 (1948).

${ }^{2}$ Felton, L. D., J. Immunol., 61, 107 (1949).

'Owen, R. D., Science, 102, 4G0 (1945). Owen, R. D., Davls, H. P. and Morgan, R. F., J. Hered., 37, 291 (1946). Stone, W., Stormont, C., and Irwin, M. R., J. An. Sci., 11, 744 (1952).

Dunsford, I., Bowley, C. C., Hutchison, A. M., Thompson, J. S. Sanger, R., and Race, R. R., Brit. Med. J., ii, 81 (1953).

${ }^{5}$ Anderson, D., Billingham, R. E., Lampkin, G. H., and Medawar, P. B., Heredity, 6, 379 (1951). Billingham, R. F., lampkin, G. H.

. Suredity, 8, $201(1952)$

' Cannon, J. A., and Longmire, W. P., Ann. Surg., 135, 60 (1952).

Billingham, R. E., Brent, L., Medawar, P. B., and Sparrow, E. M. (unpublished work).

${ }^{8}$ Billingham, R. E., Brent, L., and Medawar, P. B. (unpublished work). The passive transfer of transplantation immunity was flrst demonstrated by Mitchison, N. A., Nature, 171, 267 (1953) Proc. Roy. Soc., B (in the press).

The principle underlying this test was formulated by Chase, M. W. Abstr. 49th Gen. Meeting Soc. Amer. Bacteriol., 75 (1949).

${ }^{10}$ Billingham, R. E., and Medawar, P. B., J. Exp. Biol., 29, 454 (1952).

"Burnet, F. M., and Fenner, F., "The Production of Antibodies" (Melbourne, 1949).

${ }^{2}$ Burnet, F. M., Stone, J. D., and Edney, M., Aust. J. Exp. Biol. Med. Sci., 28, 291 (1950).

${ }^{13}$ Chase, M. W., Proc. Soc. Exp. Biol. Med., N.Y., 61, 257 (1946).

14 Mitchison (ref. 8). Medawar, P. B., Colloquia of the Ciba Foundation (in the press).

15 Summarized and reviewed by Snell, G. D., Cancer Reg 10.543 (1952).

\title{
TRANSMUTATION OF THE ELEMENTS
}

$\mathrm{T}$ RANSMUTATION of the elements, once the dream of the alchemists, is now being carried out as an everyday operation in the laboratory and on the industrial scale. The present situation and its implications, especially in so far as they concern chemistry, were reviewed in a group of three papers given before Section B (Chemistry) of the British Association at Liverpool on September 8.

In the introductory paper, Dr. R. Spence (Atomic Energy Research Establishment, Harwell) showed how transmutation is occurring continuously due to the radioactive decay of the naturally occurring radioactive elements on one hand and to the absorption of cosmic radiation by the atmosphere on the other. In the case of uranium minerals, in addition to the normal decay series the spontaneous fission of the isotope uranium-235 leads to the production of neutrons, some of which are captured by the uranium-238 isotope, which decays through neptunium-239 to plutonium. Seaborg and collaborators at the University of California have identified extremely small amounts of plutonium $(\sim 1$ part in $10^{14}$ ) in carnotite. Cosmic-ray neutrons react with the nitrogen of the atmosphere to give carbon-14 and to a smaller extent to give hydrogen-3. On the basis of data given by Libby and co-workers at the University of Chicago, it has been calculated that $16 \mathrm{mgm}$. of radiocarbon are produced in the earth's atmosphere every minute.

The development of particle accelerators and later of the chain-reacting pile has provided us with the means of bringing about transmutations on a comparatively large scale. If the construction of nuclear reactors for the generation of electrical power proceeds as might be expected, it is quite likely that at least one ton of fissile material will be consumed in
Britain per annum by 1970. World consumption might conceivably be as much as 100 tons per annum by the end of the century. The long-term future is very much dependent, however, on the successful transmutation of naturally occurring thorium or naturally occurring uranium into the fissile isotopes uranium-233 and plutonium-239, respectively, on an industrial scale. In this way the whole of the natural uranium and thorium supplies could be utilized as nuclear fuel instead of less than 1 per cent of the natural uranium as at present.

Besides the isotopes uranium-233 and plutonium239 , other isotopes of the heavy elements are produced in nuclear reactors, such as neptunium-237, americium-241 and curium-242. These were discussed in more detail by Dr. R. Hurst in the last of the three papers of the symposium. Since fission of 100 tons of nuclear fuel yields about 100 tons of fission products it is important that some effort should be made to utilize this material. The fission products consist of isotopes of elements in the middle of the Periodic System, from zinc to gadolinium. Work is going on both in the United States and in Britain to develop uses for the radiation from fission products such as for the preservation of meat and other foods, for sterilization of pharmaceuticals, for production and treatment of plastics and for bringing about chemical reactions. The fission products also contain the elements technetium and promethium, which do not occur in Nature. It is conceivable that some use will be found for these elements in due course and also for the inert isotopes arising from total decay of the active species.

The second paper, given by Dr. K. Chackett (University of Birmingham), dealt with transmutation induced by accelerated particles. The Bohr 
theory of nuclear reactions forms a useful basis for understanding the nature of the products of such reactions and also how their yield varies as a function of the energy of the bombarding particle. The cyclotron has been commonly used for the production of radio isotopes by bombardment; but there are severe restrictions on the form of target material which can be used. For example, the heat transmitted to a small area of an internal target can amount to several kilowatts. This means that only high melting point metals can be used unless the beam intensity can be reduced well below the maximum available, and that some method of cooling may be needed. Liquids can be irradiated externally, but a window is required to admit the beam on account of the fact that cyclotrons have almost always been constructed to give horizontal radiation. Examples were quoted of isotopes which can be made by means of the 60 -in. cyclotron which cannot be obtained from piles. As the product of the bombardment is usually an element different from the target element, it can generally be obtained 'carrier-free'.

Very recently, the University of Birmingham 60-in. cyclotron, in common with some other similar machines, has been used in novel ways for accelerating heavy ions such as ${ }^{12} \mathrm{C}^{6+},{ }^{13} \mathrm{C}^{6+},{ }^{14} \mathrm{~N}^{6+},{ }^{15} \mathrm{~N}^{6+}$ and ${ }^{18} \mathrm{O}^{6+}$ to energies in the region of $100 \mathrm{MeV}$. At least twelve different radioactive species have been obtained, for example, from the bombardment of aluminium. It has been known since 1951 that bombardment of uranium with ${ }^{12} \mathrm{C}^{8+}$ ions yields isotopes of the heaviest known element, californium $(Z=98)$. One of the objects of the present work at Birmingham is to see whether this work may be extended by the use of heavier bombarding particles to the production of new elements beyond californium.

Present-day researches in nuclear physics are largely concerned with obtaining particles of higher energy than can be achieved with cyclotrons. The reactions induced by protons, deuterons and alphaparticles of several hundred $\mathrm{MeV}$. given by the 184-in. synchrocyclotron at Berkeley have been studied by radiochemical means. In these reactions the bombarding particle ejects several particles from the target nucleus, a process known as spallation. A simple case appears to be the production of magnesium-28 by the reaction ${ }^{30} \mathrm{Si}(p, 3 p)^{28} \mathrm{Mg}$. This magnesium isotope has a half-life of $22 \mathrm{hr}$. and might be a valuable tracer. Although, in general, highenergy machines are unsuitable for production of radioisotopes on account of the low beam current, the high-energy proton process has some advantages over the cyclotron in this particular instance.

Even higher energies are available from synchrotrons as opposed to synchrocyclotrons; thus the Birmingham proton synchrotron has recently been put into operation and gives protons up to $1,000 \mathrm{MeV}$. The main interest in particles of such extremely high energy is in the production of mesons ; but there can be little doubt that results of radiochemical interest will also be obtained in spite of the much smaller beam currents given by such machines.

The final paper of the session was given by Dr. R. Hurst (Harwell). He pointed out that bombardment with high-energy charged particles yields at most only a few micrograms of product. It is only in the chain-reacting pile where very large quantities of neutrons can be generated and absorbed that transmutation can be brought about on a large scale. In addition to the production of plutonium in natural uranium piles, as described in the first paper, a number of isotopes of other heavy elements are produced although in much smaller quantities. For example, a long-lived isotope of neptunium (halflife $2 \times 10^{8}$ years) is produced by the reaction ${ }^{238} \mathrm{U}(n, 2 n)^{237} \mathrm{U} \stackrel{\beta}{\rightarrow}{ }^{237} \mathrm{~Np}$. A small quantity has been isolated at Harwell, and a sample containing a few milligrams was shown. Plutonium also absorbs neutrons and undergoes the following successive reactions : ${ }^{230} \mathrm{Pu}(n, \gamma)^{240} \mathrm{Pu}(n, \gamma)^{241} \mathrm{Pu}(n, \gamma)^{242} \mathrm{Pu}$. These isotopes are all long-lived $\alpha$-emitters except plutonium-241, which undergoes $\beta$-decay with short half-life to give americium-241, an isotope of element 95. So far, only a few hundred milligrams have been separated from plutonium. Americium can also be irradiated with neutrons to give americium-242, which decays by $\beta$-particle emission to give curium242 (element 96), which has been isolated in milligram quantities.

The study of the chemistry of these elements has yielded results of considerable theoretical interest. From their place in the Periodic System it might have been expected that they would resemble the transition elements. In fact, the metals are base, not noble, the oxides are refractory, the halides are slightly volatile, and the ions tend to be simple rather than complex. The properties parall ${ }^{\wedge}$ some extent, those of the rare earths. Chemical studies, X-ray spectra, absorption spectra and determinations of magnetic susceptibilities all lend support to the theory that an inner $5 f$ electron shell is being built up as one proceeds up the so-called actinide series, similar to the building up of the $4 f$ shell in the case of the rare earths. The magnetic susceptibilities of a large number of compounds of the actinide elements have been determined at Harwell, and it has, in fact, been found that UIV, $\mathrm{Pu}$ III, $\mathrm{Pu}^{\mathrm{IV}}, \mathrm{PuO}_{2}++, \mathrm{Am}$ III are paramagnetic ions obeying the Curie-Weiss law.

The solution chemistry of these elements is also of considerable interest and reaches the highest degree of complexity in the case of plutonium, where all four valence states (III, IV, V and VI) can co-exist in solution.

The mechanism and kinetics of the reactions between these various species have been worked out in considerable detail, but are not yet fully understood.

The possibility of the synthesis of still heavier elements, beyond californium, was mentioned in the second paper. Seaborg and collaborators at the University of California have made a systematic study of more than a hundred $\alpha$-emitters in the heavy element region, and it is now possible to predict both chemical and radioactive properties of elements beyond californium with reasonable certainty. It is expected that 25399 will have a half-life of the order of days, and ${ }^{252} 100$ a half-life of perhaps a few hours. Chemical separation would, therefore, be possible; but the cross-sections for the formation of such isotopes as well as the presence of competing reactions become increasingly unfavourable.

As the atomic energy industry develops, supplies of the heavier actinide elements will increase and it is likely that new and unexpected uses for them will arise. A mixture of plutonium and beryllium, for example, provides a practically permanent source of neutrons without the troublesome $\gamma$-radiation which is emitted by radium-beryllium sources. Americium has an abundant $59-\mathrm{KeV}$. X-ray, and a few milligrams have already been used experimentally at Harwell as a special radiographic source. R. SPENCE 